\title{
Testing of an Air Core Compulsator Driven 0.60 Caliber Railgun System
}

\author{
By: \\ R.L. Fuller \\ J.R. Kitzmiller \\ R.F. Thelen
}

7th EML Symposium on Electromagnetic Launch Technology

San Diego, CA

April 20-24, 1994

PR -197

Center for Electromechanics

The University of Texas at Austin

BRC, Mail Code 77000

Austin, TX 78712

(512) $471-4496$ 


\title{
Testing of an Air-Core Compulsator Driven 0.60 Caliber Railgun System
}

\author{
R.L. Fuller, J.R. Kitzmiller, and R.F. Thelen \\ Center for Electromechanics \\ The University of Texas at Austin \\ BRC, Mail Code 77000 \\ Austin, TX 78712
}

(512) 471-4496

\begin{abstract}
The Center for Electromechanics at The University of Texas at Austin (CEM-UT) is currently testing a laboratory based, small caliber, electromagnetic launcher and compensated pulsed alternator power supply. The objective of the program is to develop a compact, lightweight test bed capable of accelerating a salvo of three, 32-g masses to $2 \mathrm{~km} / \mathrm{s}$ at a rate of $10 \mathrm{~Hz}$. The 0.60 caliber, augmented railgun is powered by an $850 \mathrm{~kg}$ self-excited, air-core compulsator which develops 674 MW peak power delivered through a solid state, silicon controlled rectifier closing switch. The incremental test plan has completed testing the system at the $16,000 \mathrm{rpm}$ level with current plans to proceed to $21,000 \mathrm{rpm}$. System test data including self-excitation experiments are presented.
\end{abstract}

\section{INTRODUCTION}

The small caliber compulsator (SCC) system is the first self-excited air-core compulsator to enter testing at the Center for Electromechanics at The University of Texas at Austin (CEM-UT). The original design specifications required a lightweight, field portable machine with a high energy density. Compulsators offer high voltage and current performance in a single element energy storage device, a smooth pulse profile, and natural current zeroes which can allow low muzzle currents and simplified switching requirements. These factors enable compulsators to be extremely attractive for driving EM launchers and other pulse power devices. Typically, launch requirements dictate the type of compulsator system which is needed to accomplish the mission goals. Due to the multi-variable design problem encountered in choosing the optimum compulsator/launcher system, an optimization code is used to determine the lightest weight rotor, stator, and EM launcher which will satisfy the mission requirements. For the small caliber program, an emphasis was placed on extremely low weight and this resulted in a two-pole, self-excited, ceramic and composite rotor design [1].

The small caliber compulsator entered testing at the CEMUT in October, 1991. Subsystems of the compulsator system; the launcher, autoloader, and closing switch; had been

Manuscript received 4/20/94.

This work was supported by U.S. Army ARDEC under contract number DAAA21-87-C-0206. previously tested using the iron core compulsator [2,3]. This paper focuses on the electrical testing of the small caliber compulsator system including self-excited discharges into a laminated, series augmented launcher followed by recent improvements made to the rotor and bearing systems which will allow the compulsator to perform higher energy testing. An overview of the compulsator system design is given followed by test data representing the incremental electrical and mechanical testing of the compulsator to the $18,000 \mathrm{rpm}$ speed level. In all cases the experimental test data is compared to the predicted performance of the machine.

\section{SYSTEM DESIGN}

The SCC consists of several building blocks that define the entire system (fig. 1). The primary component in the overall system is the rotor. The SCC rotor (fig. 2) is a unique design featuring a built-up composite structure composed of 15 concentric cylinders with wall thicknesses varying from 0.25 to 0.5 in. These composite layers are interference fit over hollow silicon nitride stub shafts to form the main body of the rotor. Conductors are imbedded in two layers in the composite structure to form the armature. One layer is used to excite the field coil and the other layer is used to provide the power for launcher operation. The exterior ring on the rotor is a hoop wound UHMS graphite banding which was installed using a pressurized cure method to create a preload condition between the outer banding and the armature layer directly underneath. The conductors travel under their respective banding layers to the end of the rotor. The conductors are then split into 28 separate conductors and travel radially down the end of the rotor and are potted into place using fiberglass and epoxy. The conductors then travel under the non-thrust end bearing journal and down the brushbox shaft. The brush box shaft is a hollow titanium tube which has a fiberglass insulatory tube glued to the exterior. It is fastened to the ceramic rotor shaft using keys and a long titanium bolt through the hollow center of the ceramic shafts. Slip rings are clamped over the proper conductors to provide a current path out of the machine. Separate brush gear are used for the field coil charge path, the gun discharge path, with a common return [4].

Surrounding the rotor is the compensating shield and the field coil winding which is enclosed in the stator structure. Current is generated from the rotor via the slip rings in the end of the rotor. This current is initially developed by dis- 
charging a capacitor into the field coil generating voltage in the rotor. This ac signal is driven into a solid state rectifier box which then directs the dc power to the field coil. As the field coil power increases to a preset level, energy is directed from the rotor and transferred to the launcher by closing the solid-state gun switch. When the gun switch closes the voltage generated in the rotor armature winding is discharged into the launcher accelerating the projectile. An explosive opening switch is employed in the buswork in the event that a short develops downstream from the compulsator. The control of the explosive switch and all firing functions is provided by the firing and fault control monitor. This control box monitors the field coil charge circuitry, gun switch circuitry, autoloader circuitry, and the programmable logic controller.



(1) Compulsator rotor

(2) Field coil

(3) Field rectification circuitry

(4) Field excitation slip ring

(5) Common slip ring
(6) Discharge power slip ring

(7) Explosive opening switch (N.C.)

(8) SCR gun switch (N.O.)

(9) Launcher/Armature
Figure 1. Small caliber compulsator power circuit block diagram

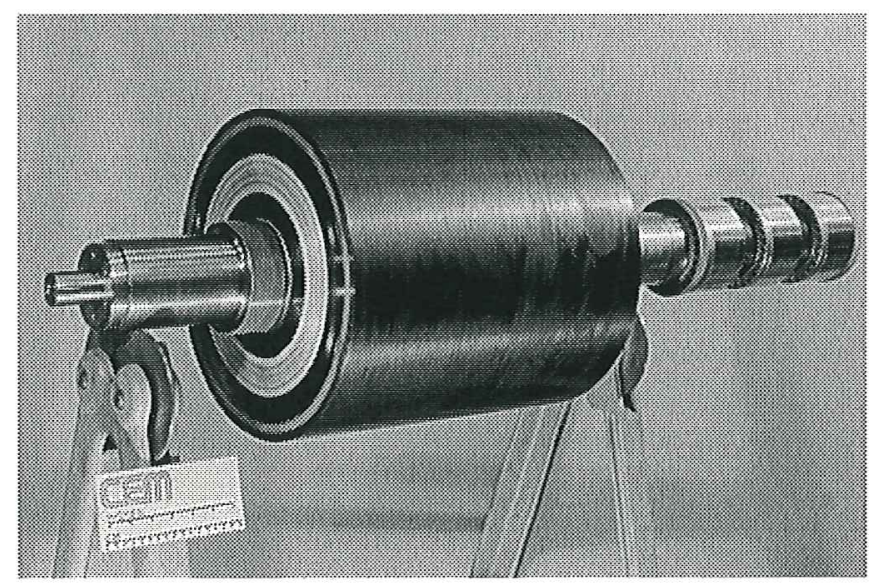

Figure 2. Small caliber compulsator rotor

\section{Testing}

Initial compulsator testing was done with an external power supply charging the field coil [4]. This was done to allow the discharge circuitry to be tested prior to bringing the self-excitation circuitry on line. A 1500 A, 500 V external supply was utilized for the initial field coil excitation. Using this power supply several low speed excitation runs were performed to calculate the important system parameters such as the various inductances and resistances of the windings and buswork components. These values were compared to the as-built predictions for major discrepancies. The values obtained from the experimental tests were then used to modify the computer simulation program. Using the simulation program a minimum safe speed of $12,000 \mathrm{rpm}$ was selected for the first discharge of the system using external field coil excitation. No significant deviations from the predicted performance from the actual test data was noted. A welcome result from the test was the open circuit voltage was approximately $6 \%$ higher than expected. This will allow the machine performance to be marginally better at a given rotational speed. The computer simulation was again adjusted to better simulate the machine performance. Due to the low energy of this test, it was difficult to simulate the performance of the armature. This was primarily due to the driving current from the compulsator shutting off well before the projectile exited from the gun. The simulation did not account for the drag effects from the armature on the rail surfaces with no driving current on the projectile. The predicted versus actual breech voltage and current from this test is shown in figure 3. This test was also used to predict the open circuit voltage developed by the field charging armature winding in the rotor. The test data concluded that at low rotational speeds, a larger seed current would be necessary to initiate the self-excitation process.

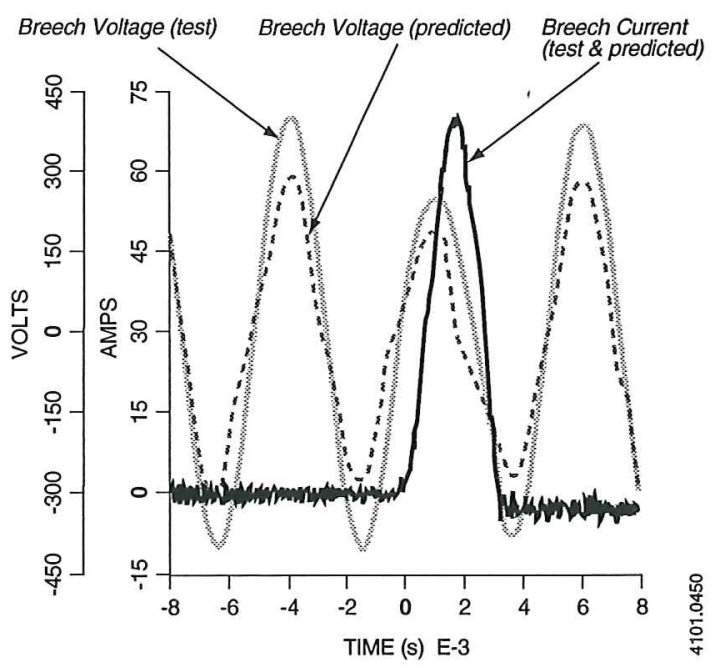

Figure 3. External excitation test data 
The next significant test was to self-excite the field coil by converting the kinetic energy in the rotor to electrical energy to charge the field coil. Prior to testing the compulsator with an EM launcher, verification of the self-excitation circuitry is required. Testing the performance of the self-excitation circuitry is crucial for proper timing and control of the compulsator discharge. To test the self-excitation circuitry, the compulsator was motored to $14,000 \mathrm{rpm}$. A seed current, provided by capacitors, was added to the field coil producing a small magnetic field. This magnetic field produced voltage in the armature winding of the machine, which produced alternating current into the rectifier circuit. The rectified current was then reintroduced into the field coil as direct current, increasing the field, thereby increasing the current and voltage from the machine (fig. 4). This process has potential for runaway and must be controlled to prevent overvoltage and overcurrent of the rectifier and field coil. Once the charge times and the control of the field charging circuit were completed, the computer simulation was modified to more closely predict the performance of the machine when discharging the machine into the EM launcher. After verifying the performance of the rectifier circuitry the EM launcher was added to the compulsator system for discharge testing.

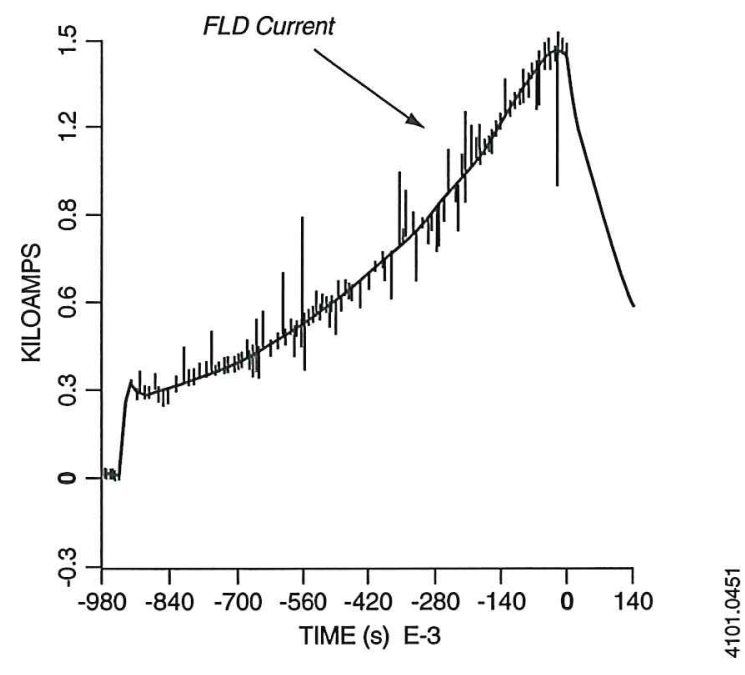

Figure 4. Self-excitation test data

The next series of tests were done in an attempt to produce the first air-core compulsator discharge into an EM launcher. The first meaningful launcher test necessitates a machine speed of 14,500 rpm. At this speed the field coil can be charged to 1,150 A creating a predicted open circuit voltage of $366 \mathrm{~V}$. The peak current is predicted to be $60 \mathrm{kA}$ into the launcher. This will accelerate an $8.6 \mathrm{~g}$ projectile to $300 \mathrm{~m} / \mathrm{s}$. The breech current and gun opening switch voltage from the actual test can be compared with the predicted values in figure 5. The open circuit voltage was measured at $389.3 \mathrm{~V}$ with a peak current of $63.2 \mathrm{kA}$ into the launcher.

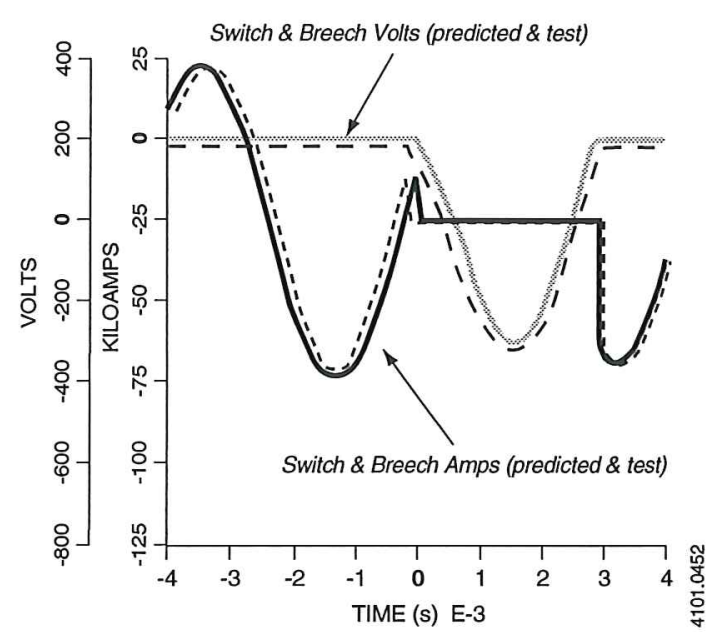

Figure 5. Self-excited compulsator discharge data predicted and experimental at $14,500 \mathrm{rpm}$

The next speed increment in the testing cycle was at the $16,000 \mathrm{rpm}$ speed level. The predicted open circuit voltage is 745 volts with a peak current of $122 \mathrm{kA}$. A $900 \mathrm{~m} / \mathrm{s}$ velocity from an $11.6 \mathrm{~g}$ projectile is predicted. The experimental data from the test indicated a rotor speed of $16,200 \mathrm{rpm}$ with an open circuit voltage of $800 \mathrm{~V}$ as seen in figure 6 . The output current from the machine reached $132 \mathrm{kA}$. The gun performance was not measured due to an instrumentation error. This test allowed the field current to reach $51 \%$ of the rated value of 5,000 A This test was a complete success with the predicted and tested performance of the machine matching extraordinarily well.

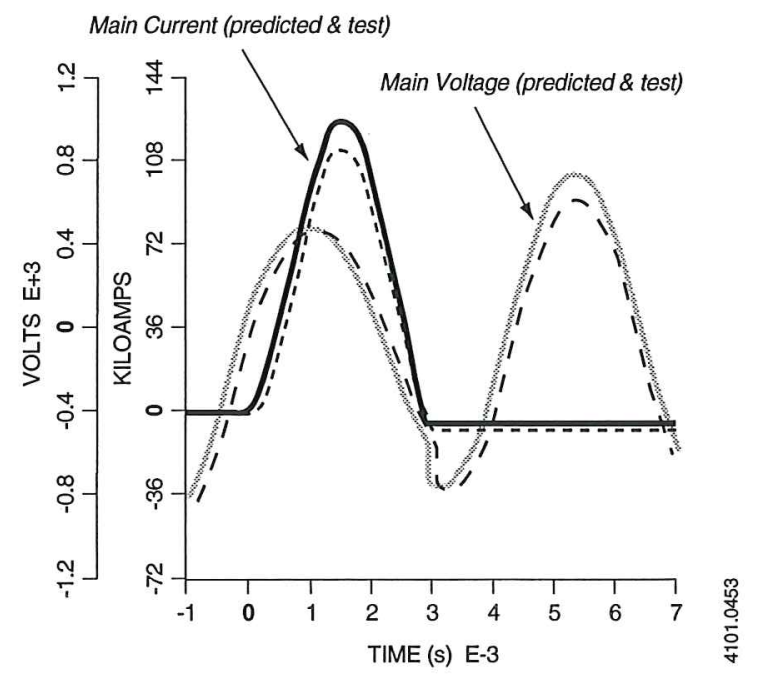

Figure 6. Self-excited compulsator discharge data predicted and experimental at $16,200 \mathrm{rpm}$. 
The next speed increment is $18,000 \mathrm{rpm}$. The predicted open circuit voltage is 1200 volts with a peak current into the launcher of $187 \mathrm{kA}$. The projectile mass is $17.718 \mathrm{~g}$ and is projected to reach $1180 \mathrm{~m} / \mathrm{s}$. Several attempts were made to spin the machine to $18,000 \mathrm{rpm}$ without success. The proximitor vibration sensing devices reached critical values and automatically caused a fault precluding electrical testing. Subsequent attempt to reach the $18,000 \mathrm{rpm}$ speed level were not successful. The speed at which the vibration sensors would fault was dropping from the $16,500 \mathrm{rpm}$ level to $12,000 \mathrm{rpm}$. It was suspected that some deterioration in the joint holding he brushbox shaft to the ceramic rotor shaft had occurred. Additional analysis and data indicated that the bearing dampers were not performing correctly. During balance testing one of the ceramic spacers in the bearings disintegrated causing damage to the thrust end bearing journal. This led to a large scale effort to refurbish the rotor and the bearing system to allow the compulsator to attain higher speeds.

A new brushbox shaft arrangement was added to the rotor to allow a stiffer joint between the titanium brushbox shaft and the ceramic rotor shaft. New journals were installed on the rotor to allow a better bearing fit. The hydrostatic dampers were changed to hydrodynamic dampers and the rotor to stator support stiffness was lowered by allowing the rotor to be supported by o-rings. A complete rotor and stator analysis was done to verify the new design was adequate for higher speeds. The rotor natural frequency was verified by rap testing the rotor in a free-free mode. The testing allowed the rotor computer model to be verified. The stator natural frequencies were analyzed using modal testing to fine tune a finite element model. After the rotor and stator were refurbished the compulsator was installed on a stiff support structure for in-situ three plane balancing. This concerted effort resulted in a successful mechanical test to $18,200 \mathrm{rpm}$.

\section{CONCLUSIONS}

The air-core compulsator has been proven as a power supply for driving EM launchers. The two-pole design of the small caliber compulsator presented interesting engineering problems in the design, fabrication, rotordynamics, and testing of the air-core machine. The testing done on this compulsator verified several key design issues important for the continued success of the compulsator as a viable power supply. The predicted performance of the machine and the actual experimental results closely correlated. The current trend in compulsator design is toward four-pole rotating field machines which will reduce the complexities encountered with the small caliber compulsator. In some applications, the two-pole machine is the highest energy density machine that can be built and the engineering problems encountered with this machine will allow better performance to be obtained from the next two-pole machine.

\section{REFERENCES}

[1.] C.W. Fulcher, et al., "Design Considerations in a 60 Caliber EM Railgun System," IEEE Transactions on Magnetics, vol 25, no. 1, January 1989.

[2.] R.L. Fuller, et al., "Design and Testing of a 0.60 Caliber, Augmented Railgun," IEEE Transactions on Magnetics, vol 27, no.1, January 1991.

[3.] R.L. Fuller et al., "Salvo Fire Experiments Using a 0.60 Caliber Electromagnetic Launcher," IEEE Transactions on Magnetics, vol 29, no.1, January 1993.

[4.] J.R. Kitzmiller, et al., "Manufacturing and Testing of an Air-Core Compulsator Driven 0.60 Caliber Railgun System," IEEE Transactions on Magnetics, vol 29, no.1, January 1993. 